\title{
THE MULTI-VEHICLE COVERING TOUR PROBLEM: BUILDING ROUTES FOR URBAN PATROLLING
}

\author{
Washington Alves de Oliveira ${ }^{1 *}$, Antonio Carlos Moretti ${ }^{1}$ \\ and Ednei Felix Reis ${ }^{2}$
}

Received April 15, 2015 / Accepted November 11, 2015

\begin{abstract}
In this paper we study a particular aspect of the urban community policing: routine patrol route planning. We seek routes that guarantee visibility, as this has a sizable impact on the community perceived safety, allowing quick emergency responses and providing surveillance of selected sites (e.g., hospitals, schools). The planning is restricted to the availability of vehicles and strives to achieve balanced routes. We study an adaptation of the model for the multi-vehicle covering tour problem, in which a set of locations must be visited, whereas another subset must be close enough to the planned routes. It constitutes an NP-complete integer programming problem. Suboptimal solutions are obtained with several heuristics, some adapted from the literature and others developed by us. We solve some adapted instances from TSPLIB and an instance with real data, the former being compared with results from literature, and latter being compared with empirical data.
\end{abstract}

Keywords: vehicle routing, covering tour problem, heuristics, urban patrolling.

\section{INTRODUCTION}

Community policing aims to serve several objectives: to gather information related to the community needs, to prevent crimes, to quickly respond to emergencies, to monitor public buildings, etc. The two most common ways of organizing patrol car operations are the allocation of a car to a certain fixed geographical location and the allocation of a car to a certain route covering a larger area. The second is the method usually chosen by the municipal guards and military police in the state of São Paulo, Brazil. The planning of these routes rarely adopts a scientific approach, empirical rules are used instead.

One of the most important features of community policing is the contact between the members of the community and the patrolling officers, improving the exchange of information between

\footnotetext{
*Corresponding author.

${ }^{1}$ Faculdade de Ciências Aplicadas, Universidade Estadual de Campinas, 13484-350 Limeira, SP, Brasil.

E-mail: washington.oliveira@fca.unicamp.br; moretti@ime.unicamp.br

2 Departamento de Matemática, Universidade Tecnológica Federal do Paraná, 84016-210 Ponta Grossa, PR, Brasil.

E-mail: edneif@utfpr.edu.br
} 
the community and the police force. Balanced routes with respect to the number of visits can improve the whole process of community patrolling, since this can reduce the number of persons and visits per officer.

Using a fleet of known size, we consider how to efficiently construct routes in order to patrol a given geographical area. The routes should satisfy the following criteria: a certain set of sites must be included in the routes; a second set may or may not belong to the routes; a third set is made up of sites that must be observed (covered) by the patrolling officer, in the sense that these sites are not visited, but they must be close enough to at least one visited site; the number of routes must equal the number of available vehicles; the routes must be balanced, that is, the number of visited sites for each route is approximately the same; all routes must start and finish at the same geographical point, the base of operations.

The problem is modeled as an integer programming problem. The model presented in this paper is related to the multi-vehicle Covering Tour Problem ( $m$-CTP), where we discard the vehicle capacity constraints and include a certain balance among the vehicles. The resulting combinatorial optimization problem is NP-complete, which justifies the use of heuristics developed in this paper to obtain suboptimal solutions of acceptable quality in reasonable time.

The heuristics were implemented in MATLAB ${ }^{\circledR}$ and compared using several instances from TSPLIB. These instances, together with real data from the city of Vinhedo, São Paulo, Brazil, were used to validate the code.

In Section 2, we define the balanced multi-vehicle urban patrolling problem. The mathematical formulation is described in Section 3. The heuristics developed in this paper are presented in detail in Section 4. In Section 5, we present the numerical experiments. Finally, the conclusions are given in Section 6.

\section{BUILDING ROUTES FOR URBAN PATROLLING}

In the problem considered in this paper, a set of geographical points that need to be visited during a routine patrol was determined by the police force, which might include schools, hospitals, public buildings, etc. Additionally, there is another set that must be at convenient distance from the route, for instance, public parks, community centers, bank agencies, etc. Good designs of routes are of crucial importance, due to the limited resources (the size of the fleet) available to cover usually large geographical areas. Therefore, the patrolling officers must be guided from one visit to another in order to avoid bad empirical circuits.

More specifically, we will build routes for urban patrolling for the city of Vinhedo. This city is located approximately $80 \mathrm{~km}$ east of the city of São Paulo, Brazil. Its economy is mostly based on agriculture, in particular the growing of grape and production of related goods, specially wine. It occupies an area measuring roughly $81.742 \mathrm{~km}^{2}$, and, as of 2010, had about 63,500 inhabitants. It is part of a group of cities known as the fruit circuit, which promotes three to four festivals per year, the fig festival, the strawberry festival, for example. The per capita income is high and the criminality rate is low. 
The Municipal Guard of the city of Vinhedo must assist the population in public safety matters, crime prevention being the main objective to be achieved by the police force. Preventive measures that increase the contact between the patrolling officers and members of the community are of fundamental importance to community policing (NEV/USP, 2009). This strategy aims to build a partnership between the community and Municipal Guard based on the premise that a collective effort must be made to improve public safety. This means that the people in a certain area have to not only participate in the discussions about safety and establish priorites and strategies, but also share with the officers that patrol this area the responsibility for the safety of the region. We can highlight the following measures: organization of public audiences to discuss the community problems in order to develop strategies and priorites; mobilization of the community for self-protection and solving problems that generate crimes; motivate the frequent dialogue between the officers and the community.

Ideally, the patrolling officers must stop in each visit point, interact with the people there, and watch certain points of interest within viewing distance. Aiming a greater proximity with the community, the chief of operations needs to designate a balanced set of visits for the patrolling cars, reducing the number of persons per officer. However, in practice, the city is divided into sectors (see Fig. 3), and a certain number of vehicles is assigned to a given sector. Moreover, the patrolling officers make the circuit of visits empirically, based on their knowledge of the geographical area, which may be unproductive. Figure 3 illustrates the distribution of five sectors within the Vinhedo city region, denoted by A, B, C, D and E. Note that the geographical points are not equally distributed within these sectors.

There are regions where the geographical points of interest are more concentrated than others, due to the characteristics of a geographical area (e.g., the distances between two schools may be much larger in peripheral areas than in central ones). Consequently, if we obtain balanced routes, there might be shorter routes in the central regions than in peripheral ones. Therefore, the fact that there are routes which are shorter than others is not inconsistent with our proposal for building routes for urban patrolling, since the efficiency of the contacts established by the patrolling officers with the members of the community outweighs the balance among the lengths of routes.

In our approach, we aim to build routes in the context urban patrolling that balance the number of visits without a prior division of the geographical area into sectors. The set of visits for each patrolling car is decided by dividing the set of all visits into clusters. Once the set of visits for a patrolling car is obtained, to determine the order to the visits, we have chosen to minimize the overall length of all routes instead of choosing the order empirically. Thus, the problem is modeled as an integer programming problem whose the main objective is to determine balanced circuits in number of visits for each patrolling car.

A proper survey of the features of the problem was conducted with Vinhedo patrol professionals. Ideally the distances (or travel times) among geographical points would be calculated separately from real data, taking into account possible paths between nodes, average speeds, etc. This would imply in non-symmetrical distances. Nevertheless, in the real instance considered, involving the 
city of Vinhedo, the true path lengths (corresponding to routes using the city streets) were not known. Therefore, the Euclidean distance was chosen as an approximation to determine each route. Note that once the order of visits is established, the patrolling officers can use their experience to decide how to go from one visit to the next.

There are similarities between the design of routes for urban patrolling and the model presented in this paper, namely, the construction of circuits covering certain geographical points, while visiting mandatory and optional ones. The number of visits is balanced and the number of routes is fixed (we do not want cars and personnel idling at the base). Throughout the text we present our model, and more importantly, our strategy to obtain approximate solutions.

The model involves a graph $G=(V \cup W, E)$, whose nodes correspond to strategic geographical points, e.g., intersections, certain locations, etc., that are either important on its own, or serve to establish reference locations for the routes. The node set is partitioned into two subsets: $V=$ $\{0, \ldots, n\}$ is the set of nodes that may belong to routes and $W=\{n+1, \ldots, n+\ell\}$ is the set of nodes that must be covered, but not visited. The set $V$ contains a subset $T$ of nodes that must be visited. Node $0 \in T$ corresponds to the base. The set $V \backslash\{0\}$ is denoted by $V^{*}$. The symbol $T^{*}$ denotes the set $T \backslash\{0\}$.

The set $E$ contains all possible undirected arcs between nodes of $V$. The entry $c_{i j}$, of the distance matrix $C=\left(c_{i j}\right)$, contains the Euclidean distance between nodes $i, j=0,1, \ldots, n+\ell$. This assumption implies that $C$ is symmetric with zero diagonal. In this case, we choose to represent the set (undirected $\operatorname{arc}$ ) $\{i, j\}$ by the ordered pair $\left(i^{*}, j^{*}\right)$, where $i^{*}=\min \{i, j\}$ and $j^{*}=\max \{i, j\}$. The size of the fleet is denoted by $m$ and the admissible distance from a node in the route to a node that must be covered is denoted by $c$. We need one last parameter, to express our tolerance regarding the lack of balance between different routes. We compare routes by means of the number of nodes each route contains. The number $r$ denote the maximum difference allowed for the total visited nodes in any two routes.

We want to construct $m$ routes satisfying the following conditions:

- Each route is a circuit in $G$ containing node 0 .

- Each node in $V^{*}$ must belong to at most one route.

- Each node in $T^{*}$ must belong to exactly one route.

- There must be at least a visited node at a distance of at most $c$ from each node in $W$.

- The number of nodes in any two routes differ by at most $r$.

Variants of this model have been used in many contexts. Labbé \& Laporte (1986) discuss how to simultaneously decide on the location of mailboxes and the planning of routes involving candidate sites, such that all users are close enough to some mailbox. The planning of routes for medical mobile units (Brown \& Fintor, 1995; Foord, 1995; Hodgson et al., 1998; Oppong \& Hodgson, 1994; Swaddiwudhipong et al., 1995) bears many similarities with the planning of 
urban patrol routes. In places where medical services for small villages are rendered by mobile units, their routes must be planned in such a way that, in addition to visiting specific locations, the villages not included in the tour must be within walking distance from some other village included in the tour. The model can also fit situations in business sectors, see Simms (1989) for an application in dairy practice.

The special case of $m$-CTP where $V=T$ reduces to a vehicle routing problem (Laporte, 1992) with unit demand. Baldacci et al. (2005) present three Scatter Search heuristic algorithms for the 1-CTP. Motta et al. (2001) use a GRASP approach to solve a variant of the 1-CTP, the Generalized Covering Tour Problem, whose minimum length tour may pass through a subset of $W$. In another point of view, Jozefowiez et al. $(2004,2007)$ present a multi-objective covering tour problem, a generalization of the 1-CTP where the parameter $c$ is omitted and replaced by an objective. They propose a hybrid strategy approach that combines a multi-objective evolutionary algorithm with a branch-and-cut algorithm to determine the Optimal Pareto sets.

Hà et al. (2013) obtained exact solutions for a variation of $m$-CTP, where the constraint on the length of the routes is relaxed. They used a branch-and-cut algorithm and a metaheuristic (based on the evolutionary local search) to obtain those solutions. Jozefowiez (2014) and Lopes et al. (2013) used a branch-and-price algorithm, in which a column generation approach is applied at each node of the search. Despite the recent applications for $m$-CTP using variations of the exact methods branch-and-bound, branch-and-cut, and branch-and-price, we did not succeed in solving large instances. In our application, in the context of urban patrolling, the instances are of the order of more than two thousand points.

\section{MATHEMATICAL FORMULATION OF THE PROBLEM}

In order to facilitate the modeling of the covering restrictions, we define the set of nodes in $V$ within the allowed prescribed distance $c$ from each node $j \in W: S_{j}=\left\{i \in V \mid c_{i j} \leq c\right\}$. We may suppose without loss of generality that $\left|S_{j}\right| \geq 2$, since if there is only one node $i \in V$ close enough to some $j$, then we may as well include $i \in T$ and eliminate $j$ from $W$. Similarly, we may assume that $S_{j} \cap T=\emptyset$, for all $j$, since we do not need to worry about covering nodes that are close enough to some node in $T$. With these assumptions, $S_{j}=\left\{i \in V \backslash T \mid c_{i j} \leq c\right\}$ and its cardinality is at least two, for all $j \in W$.

The model contains two sets of binary variables. The variable $y_{i k}$ is 1 if node $i$ is visited by vehicle $k$, and 0 otherwise, for $i \in V, k=1, \ldots, m$. The variable $x_{i j k}$ is 1 if vehicle $k$ uses arc $(i, j)$ in its route, and 0 otherwise.

$$
\begin{array}{ll}
\text { Min } & \sum_{k=1}^{m} \sum_{i=0}^{n-1} \sum_{j=i+1}^{n} c_{i j} x_{i j k}, \\
\text { s.t. } & \sum_{k=1}^{m} \sum_{i \in S_{j}} y_{i k} \geq 1, \quad j \in W,
\end{array}
$$




$$
\begin{aligned}
& \sum_{k=1}^{m} y_{i k} \leq 1, \quad i \in V \backslash T, \\
& \sum_{k=1}^{m} y_{i k}=1, \quad i \in T^{*}, \\
& \sum_{i=0}^{h-1} x_{i h k}+\sum_{j=h+1}^{n} x_{h j k}=2 y_{h k}, \quad h \in V^{*}, \quad k=1, \ldots, m, \\
& \quad \sum_{i \in S, j \in V \backslash S} x_{i j k} \geq 2 y_{h k}, \quad S \subset V^{*}, h \in S, \\
& \sum_{i=1}^{n} x_{0 i k}=2, \quad k=1, \ldots, m, \quad 2 \leq|S| \leq n-1, k=1, \ldots, m, \\
& \sum_{i=1}^{n} y_{i p}-\sum_{i=1}^{n} y_{i q} \leq r, \quad p, q=1, \ldots, m, \\
& \sum_{i=1}^{n} y_{i p}-\sum_{i=1}^{n} y_{i q} \geq-r, \quad p, q=1, \ldots, m, \\
& y_{0 k}=1, \quad k=1, \ldots, m, \\
& y_{i k}, x_{i j k} \in\{0,1\}, \quad i, j \in V, \quad k=1, \ldots, m .
\end{aligned}
$$

Using these variables, the "cost" of a solution, given in (1), is the cumulative length of all routes, which we wish to minimize. The constraints are modelled as follows. The covering of node $j \in W$ is guaranteed by (2). Constraints (3) make sure that each node in $V \backslash T$ belongs to at most one route. The fact that every node in $T$ must be visited by some tour is expressed in (4). Constraints (5) imply that, if node $h$ belongs to route $k$, then it has two neighbors in the route. Constraints (6) avoid subtours, by forcing that, if node $h \in S \subseteq V^{*}$ belongs to route $k$, then the cut-set $(S, V \backslash S)$ must contain at least two arcs of route $k$. (7) guarantees that each route has two arcs incident to the base. The maximum difference between the number of nodes of different routes is enforced by (8) and (9), i.e., the maximum difference

$$
\tilde{r}=\max _{1 \leq p, q \leq m}\left|\sum_{i=1}^{n} y_{i p}-\sum_{i=1}^{n} y_{i q}\right|
$$

obtained in the solution must be less than or equal to the parameter $r$. Constraints (10) force that the base belongs to every route. The last set of constraints, (11), simply specifies the allowed values for the variables.

\section{HEURISTICS}

The heuristics developed for the balanced multi-vehicle urban patrolling problem modeled in the last section are divided into three phases. In Phase 1 , subsets $V_{k}$ and $W_{k}, k=1, \ldots, m$, 
are selected, where $V_{k}$ and $W_{k}$ are the nodes that may be visited and that should be covered by route $k$, respectively. Phase 2 deals with $m$ 1-CTP problems, defined on the subgraph induced by $V_{k} \cup W_{k}$. At this point, $m$ closely related problems are considered separately. The last phase tries to improve the solution by taking this interrelation into account. The three-phase sequence is repeated according to a criterion specific to the routine employed in Phase 1. The best solution in the whole loop of three phases (or outer iterations) is selected.

Table 1 below summarizes the routines employed in each phase of the various heuristics. The routine employed in Phase 1 will lend its name to the heuristic. Note that they share Phase 2, and the first three heuristics use Balanced 2-opt in Phase 3, while the Sector Partition uses Multicover Elimination.

Table 1 - Routines according to Phase.

\begin{tabular}{|c|c|c|}
\hline Phase 1 & Phase 2 & Phase 3 \\
\hline Greedy & \multirow{8}{*}{$\begin{array}{l}\text { Modified } \\
\text { 1-CTP } \\
\text { Routine }\end{array}$} & \multirow{6}{*}{$\begin{array}{l}\text { Balanced } \\
\text { 2-opt }\end{array}$} \\
\hline Selection & & \\
\hline Sweep & & \\
\hline Routine & & \\
\hline Route-first/ & & \\
\hline Cluster-second & & \\
\hline Sector & & Multicover \\
\hline Partition & & Elimination \\
\hline
\end{tabular}

Our mathematical model resembles the model in Hachicha et al. (2000). However, in that model the length and number of visits per tour are limited and the number of routes is variable, whereas in this model the number of visits is balanced and the number of routes is fixed. In order to take into account these differences, we consider modified versions of the sweeping algorithm, Route-first/Cluster-second algorithm and 2-opt* algorithm presented in their paper. The Sweep Routine corresponds to steps 1 and 2 of the sweeping algorithm. Similarly, Route-first/Clustersecond is formed by steps 1 and 2 of the algorithm of same name. The Balanced 2-opt routine contemplates improvements via arc swapping, and is adapted from the 2-opt* algorithm.

The Modified 1-CTP Routine is a modification of the heuristic described in Gendreau et al. (1997) for the covering tour problem. The remaining routines were developed by us.

The Greedy Selection routine gradually selects sites using a criterion that selects the nearest site to the one previously selected, forming a circular ordered list. The nodes in this list are then partitioned into $m$ subsets of approximately equal size, keeping the order of the original list and starting with the first node in the list. In subsequent iterations, the selection step is not repeated. Instead, we simply shift by one the order of the nodes in the list and redo the partitioning. This is repeated approximately $t / m$ times, where $t$ is the cardinality of the list.

In the Sector Partition routine, we use a geographical approach to divide $V, T$, and $W$, into $m$ subsets, corresponding to circular sectors. Each outer iteration corresponds to a counterclockwise 
shift of the sectors. This simple geographical division is used to reduce the computational time in Phase 1.

The Multicover Elimination checks whether some node in $W$ is covered by more than one node included in a route. If this is the case, there may be room for improvement, by removing one of the superfluous nodes.

In the described routines, it is often necessary to know the subset of nodes in $W$ covered by a particular node in $V$. The subset covered by node $i$ is denoted by $C_{i}=\left\{j \in W \mid i \in S_{j}\right\}$. Recall that $S_{j}=\left\{\ell \in V \backslash T \mid c_{\ell j} \leq c\right\}$.

\subsection{Greedy Selection}

Initially we form a single route $R=\left(h_{0}, h_{1}, \ldots, h_{z}\right)$ that contains all nodes in $T$ and covers all nodes in $W$ as follows. The routine starts with $h_{0}=0, R=\left(h_{0}\right)$ and $L=T^{*} \cup W$. The set $L$ is gradually emptied using the criterion that selects the nearest site to the one previously selected in $L$. If the chosen node $h$ belongs to $T$, it is simply appended to $R$, and $\{h\} \cup C_{h}$ is removed from $L$. If $h$ belongs to $W$, then one selects from $S_{h}$ the node that covers the greatest number of yet uncovered nodes, say node $\ell$, and appends it to $R$. Then $C_{\ell}$ is removed from $L$.

In the next two paragraphs, we detail the main differences between our approach and Hachicha's Sweep and Route-first/Cluster-second routines. There, the selection of the sets $V_{k}$ and $W_{k}, k=$ $1, \ldots, m$, is made in order to minimize the number of routes, while satisfying the demand, the capacity of the vehicle and their constraints on the length of the routes. In our approach, we choose $V_{k}$ and $W_{k}$ with approximately the same number of elements to keep the balance among routes.

Here, and in the next two routines, once $R$ is constructed we consider the sequence $R^{*}=R \backslash\{0\}$ as a circular list. In order to apply Phase 2, we need to divide $V, T$, and $W$ into $m$ subsets that are induced by a partition of $R^{*}$ as follows. Let $p=\lfloor z / m\rfloor, q=z-m p$, where $z$ denotes the number of nodes in $R^{*}$. Starting from the first node in $R^{*}$, we select $q$ subsets of sequential nodes of size $p+1$, and $m-q$ subsets of size $p$. The nodes in $V$ (resp., $T$ ) in the $k$ th subset union $\{0\}$ are denoted by $V_{k}$ (resp., $T_{k}$ ). The $k$ th subset of $W$ is $W_{k}=\bigcup\left\{C_{i} \mid i \in V_{k}\right\}$.

In the next round, the partition of $R^{*}$ will start at the second node, and then third, and so on, in a total of $p$ or $p+1$ outer iterations, depending on whether $z$ is a multiple of $m$ or not.

\subsection{Sweep Routine}

The difference between this routine and the previous one is the strategy used to build the single route $R=\left(h_{0}, h_{1}, \ldots, h_{z}\right)$. The sweeping process is applied to the vertices $T \cup W$ and several solutions are generated. The routine starts with $h_{0}=0, R=\left(h_{0}\right)$ and $L=T^{*} \cup W$. Choose an arbitrary node $\bar{h} \in L$ and consider a half line from $h_{0}$ passing through $\bar{h}$. The set $L$ is gradually emptied using the criterion that sweeps the nodes $h \in L$ according to the ascending order of the angles $\theta_{h}=\widehat{\bar{h} h_{0} h}$. If the chosen node $h$ belongs to $T$, it is simply appended to $R$, and $\{h\} \cup C_{h}$ 
is removed from $L$. If $h$ belongs to $W$, then one selects from $S_{h}$ the node that covers the greatest number of yet uncovered nodes, say node $\ell$, and appends it to $R$. Then $C_{\ell}$ is removed from $L$. Once $R$ is constructed, the process continues as in the Greedy Selection routine.

\subsection{Route-first/Cluster-second}

Again, the difference between this routine and Greedy Selection routine is the strategy that we build the single route $R=\left(h_{0}, h_{1}, \ldots, h_{z}\right)$. Here, a feasible 1-CTP solution for $V, T$, and $W$ is determined by means of the Modified 1-CTP Routine (section 4.5), say route $R=$ $\left(h_{0}, h_{1}, \ldots, h_{z}\right), h_{0}=0$, that contains all nodes in $T$ and covers all nodes in $W$. This tour is then divided into smaller feasible routes as in the Greedy Selection routine.

\subsection{Sector Partition}

This routine is applicable only in cases where there is a geographical model of the problem. The site associated with the base node is taken as the geographical center of a circular disk containing all locations (nodes) under consideration. This disk is partitioned into $m$ circular sectors of same central angle. Nodes corresponding to sites in the $k$ th partition form the sets $V_{k}, T_{k}$, and $W_{k}$. The first partition is arbitrary. In the next iteration, the sectors are shifted counterclockwise by $360^{\circ} / t$, and this is repeated until we return to the original partition, a total of $t$ outer iterations. In the computer experiments, the value $t=10$ was used. Due to its simplicity, if the distribution of nodes is non-uniform, this way of choosing the partitions $V_{k}, T_{k}$, and $W_{k}$ does not guarantee the equilibrium among the number of nodes in each partition, possibly producing unbalanced routes.

\subsection{Modified 1-CTP Routine}

Gendreau et al. (1997) developed a heuristic for the covering tour problem (1-CTP), using elements of the GENIUS heuristic for the traveling salesman problem (TSP) of Gendreau et al. (1992) and PRIMAL1 set covering heuristic of Balas \& Ho (1980).

This heuristic uses the fact that, if the set of nodes that should be visited (that is, the support of the optimal $y$ ) is known, then the covering tour problem reduces to a traveling salesman problem. This suggests the construction of a covering problem by considering the "covering" aspect separately, namely the combinatorial optimization problem (12) below. Notice that, since $m=1$, there is no need for the tour index $k$.

$$
\begin{array}{ll}
\text { Min } & \sum_{j \in V} c_{j} y_{j}, \\
\text { s.t. } & \sum_{j \in S_{i}} y_{j} \geq 1, \quad i \in W, \\
& y_{j}=1, \quad j \in T, \\
& y_{j} \in\{0,1\}, \quad j \in V .
\end{array}
$$


In PRIMAL1, variables $y_{j}$ are gradually included in the solution according to a greedy rule, using one of three merit functions: (i) $f\left(c_{j}, b_{j}\right)=c_{j} /\left(\log _{2} b_{j}\right)$; (ii) $f\left(c_{j}, b_{j}\right)=c_{j} / b_{j}$; (iii) $f\left(c_{j}, b_{j}\right)=c_{j}$; where $b_{j}$ is the number of uncovered (in the current partial solution) nodes in $W$ that are covered by $j$, and $c_{j}$ is the cost to insert $j$ in the current solution.

On the other hand, TSP routes are also constructed incrementally in GENIUS. During the construction phase, a tentative tour is built starting with three arbitrarily selected nodes and using a general insertion procedure (GENI), with rules for selecting candidates and rules for evaluating the inclusion in the tour. Once a complete tour is obtained, one seeks to improve it by removing and reinserting each node of the tour, in a post-optimization procedure called US (for Unstringing and Stringing).

GENIUS and PRIMAL1 are combined to produce a covering tour heuristic as follows. Nodes are gradually selected and added to the set of nodes that should be visited, and a new approximate solution to the TSP involving each insertion of these nodes is obtained using GENIUS. This procedure stops when all nodes in $W$ have been covered. The selection of the each node is made by considering the merit functions in PRIMAL1 heuristic using the coefficient $c_{j}$ (cost to insert this node in the approximate solution to the TSP, calculated by GENI selection rules). At the end, post-optimization is applied by removing superfluous nodes. The whole process is done for each merit function, two sequences of the merit functions (i)-(ii)-(iii) and (i)-(iii)-(ii) are considered, and the best solution is selected.

Numerical experiments with our code for the various heuristics showed that the GENI part was fast and produced acceptable results, whereas the post-optimization US routine was quite costly. After several trials, we arrived at a modified routine of Gendreau et al.'s covering tour heuristic, in order to avoid the repeated construction of unnecessary approximate solutions of the TSP at the insertion and removal of each node. This is done by inserting the new node using GENI rules and removing superfluous nodes using US rules without discarding the previous TSP solution.

More details about the experiments with the different heuristics tried out, which showed a better computational performance with these modifications, can be found in Oliveira (2008). In the modified routine listed below, the merit functions are used in the sequence (i)-(ii)-(iii).

STEP 1. Let $H \leftarrow T, \bar{z} \leftarrow \infty$. Let $f$ be the merit function specified in (i). Let $z$ be the value of the tour obtained applying GENIUS to the TSP involving the nodes in $H$. If all nodes in $W$ are covered, go to Step 2. Otherwise, go to Step 3.

STEP 2. If $z \leq \bar{z}$, let $\bar{z} \leftarrow z, \bar{H} \leftarrow H$. If the definition used for the merit function is the last, stop with the best solution so far, with value $\bar{z}$ and set of visited nodes $\bar{H}$. Otherwise, remove from $H \backslash T$ nodes associated with multiple covered nodes in $W$ using US. Go to Step 3 with the next definition of the merit function $f$.

STEP 3. Select within $V \backslash H$ node $h^{*}$ using PRIMAL1, with cost coefficients $c_{j}$ calculated using criteria defined in GENI. Insert $h^{*}$ in the route employing GENI, and let $H \leftarrow$ 
$H \cup\left\{h^{*}\right\}$. Repeat the process until all nodes in $W$ are covered. Let $z$ be the value of the tour obtained. Go to Step 2.

When this routine is used in Phase 2, it is run $m$ times with the sets $V_{k}, T_{k}$, and $W_{k}$, for $k=1, \ldots, m$, constructed in Phase 1 .

\subsection{Balanced 2-opt}

This is a modification of the 2-opt* heuristic. The changes introduced aim to keep the balance, in terms of number of nodes, among the routes. Steps 1 and 2 are adaptations, since in their approach, the number of the routes can decrease and changes between arcs are allowed provided the length of the routes and number of visits are within the preset values, while in our approach, those changes can be made only if the balance between routes is not destroyed and the number of routes is not altered (we do not want cars and personnel idling at the base). Step 3 is different, it considers swapping of nodes belonging to different routes. Notice that the exchanges considered in this last step do not alter the number of nodes in each route.

At this point, the initial set of $m$ routes constructed in Phase 1 has already undergone improvement in Phase 2, and the following procedure is executed.

STEP 1. Let $\rho$ be the smallest number of nodes per route, excluding the base node. Transform the set of $m$ routes into a single route by replacing the base node by $m$ artificial copies of the base node (see Fig. 1). Make a list of all possible pairs of distinct arcs in this single route.
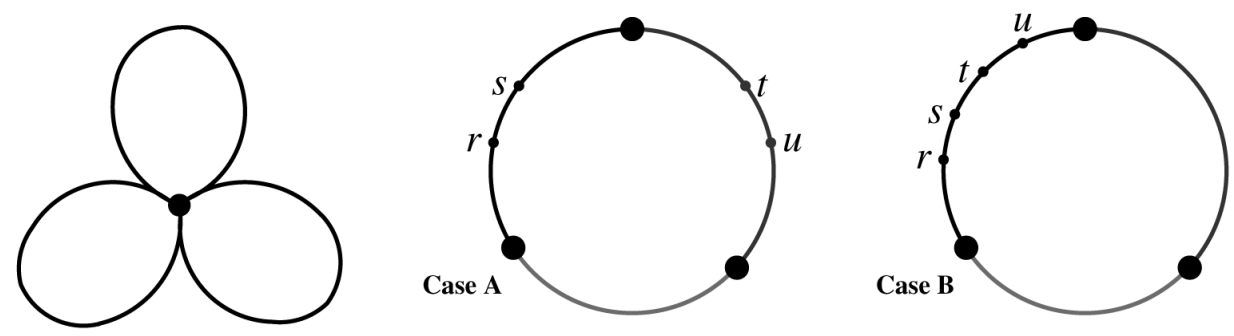

Figure 1 - How to build a single route for $m=3$, and two patterns of arc-pairs.

STEP 2. In this step, the arc-pairs in the list are considered sequentially, until either a better solution is reached and return to Step 1 or there is no pair left, continue with Step 3. Let $\{\{r, s\},\{t, u\}\}$ be the current pair. Consider the two possibilities of replacement: (i) $\{\{r, t\},\{s, u\}\}$, (ii) $\{\{r, u\},\{s, t\}\}$. The replacement may split the single route into two routes (see Fig. 2). If this happens, the new routes correspond to a feasible solution only if each of them contains at least one copy of the base node. Furthermore, the number of nodes in the route(s) between each pair of copies of the base node (or in the case where one of the subroutes created by the replacement contains only one copy of the base node) 
must be at least $\rho$. For each feasible solution, calculate its objective value. If there is an improvement, recover $m$ routes from the best improved single (or pair of) route(s) and go back to Step 1. If the end of the list is reached without (feasible) improvement, recover $m$ routes from the best improved single (or pair of) route(s) and go to Step 3.

Case A1
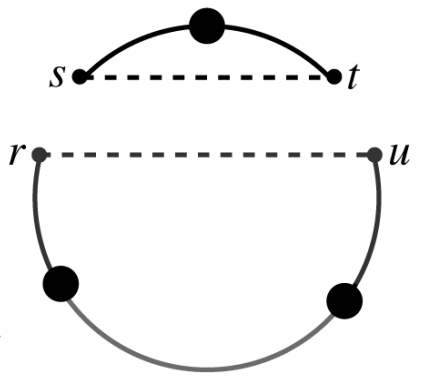

Case A2

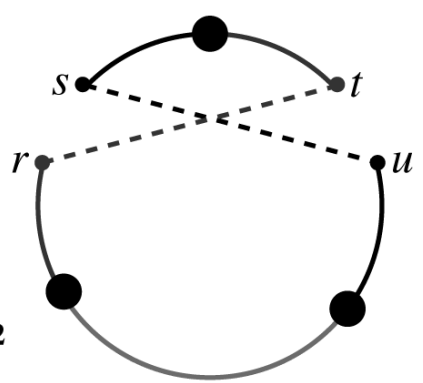

Case B1

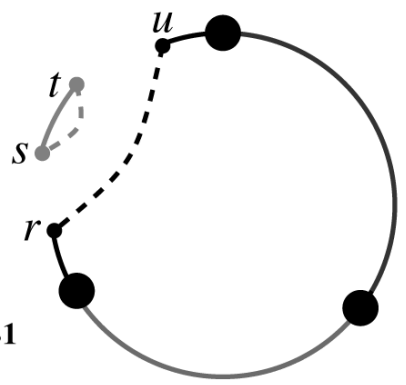

Case B2

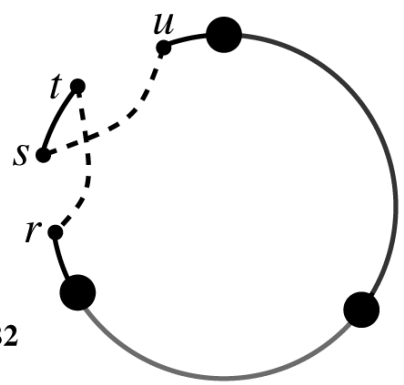

Figure 2 - Two cases of replacement of arc-pairs (Cases A and B in Figure 1).

STEP 3. Make a list of pairs of nodes, each distinct from the base node, and belonging to different routes. Consider the pairs sequentially. For each pair, calculate the value of the alternative solution obtained by swapping the nodes. Keep the solution with best objective function value.

In Step 1 of the Balanced 2-opt, we transform the set of $m$ routes into a single route (Fig. 1) in the same manner as described in Lenstra \& Rinnooy Kan (1975). Case A and Case B in Figure 1 are two possible configurations of arc-pairs in distinct routes and in the same route, respectively. The choice of $\rho$ as the smallest number of nodes per route, and the fact that of the arc-pair exchanges in Step 2 produces routes with at least $\rho$ nodes guarantees the balance among routes.

The exchanges of arcs in Step 2, 2-opt moves, are particular cases of $k$-opt move algorithm (LK) developed by Lin \& Kernighan (1973). In Step 3 the swapping of nodes between different routes can be considered as a 4-opt move, since the two arcs that share each exchanged node are deleted and replaced by four new arcs. Although the extension and generalization LKH-2 algorithm developed by Helsgaun (2009) greatly improves LK, we chose 2-opt and 4-opt moves, since they have a reasonably good performance and the balance constraints can be easily checked. 
Several patterns resulting from $k$-opt moves are not suitable to our model, since they destroy the balance and create disconnected subroutes. Figure 2 (as in Hachicha et al. (2000)) illustrates some examples of configurations after the replacement of arc-pairs, where four cases are obtained according to the relative position of each arc-pair, namely the arc-pairs being either in distinct routes (Cases A1 and A2) or in the same route (Cases B1 and B2).

In Case A1, the two disconnected routes obtained are feasible once the balance constraints are satisfied. Note that this exchange is not allowed in the $k$-opt moves. In Case A2, one can produce unbalanced solutions depending on the number of nodes between the copy of the base and an arcpair node. Case B1 is infeasible since one chain does not contain a copy of the base. Finally, in Case B2, there is a change in the order of nodes in a route, which may improve the solution.

\subsection{Multicover Elimination}

If a node in $W$ is covered by more than one node, we may improve the solution by eliminating one of the superfluous nodes. At this point, the initial set of $m$ routes constructed in Sector Partition has already undergone improvement in Phase 2. Note that only nodes in $V \backslash T$ (the support of $y$ that are not in $T$ ) may be removed from the solution. If a node $i$ may be removed, we consider the alternative solution obtained by deleting the arcs incident to $i$ and adding the arc linking its neighbors. Observe that, since the distances between nodes satisfy the triangle inequality, the removal of a node always implies in the decrease of the objective function.

The possibility of removal of node $i$ is checked in the brute force way: we delete it from the route and check whether this results in some node in $W$ being uncovered. This is a two-step procedure. In the first step we build a list of candidates for removal, and in the second step we examine the list sequentially, trying to remove as many nodes as possible.

STEP 1. Examine all nodes in the route that belong to $V \backslash T$ and build a list of superfluous nodes in descending order with respect to the amount of decrease in the objective function implied by their removal.

STEP 2. Consider the nodes in the list sequentially and remove the nodes whose removal does not destroy the required coverage and balance among routes.

Note that this procedure is simpler than the Balanced 2-opt routine and it was chosen as the postoptimization for Sector Partition heuristic in order to reduce the computational time in Phase 3.

\section{NUMERICAL EXPERIMENTS}

The heuristics were tested on a set of adapted instances from TSPLIB and on an instance constructed from official data of the city of Vinhedo, São Paulo. They were implemented in MAT$\mathrm{LAB}^{\circledR}$ and tests were run on a PC with an Intel i7-4790S $3.20 \mathrm{GHz}$, with 8 GB of RAM DDR3 and Linux operating system. The characteristics of the adapted instances are described next, followed by comments about the collection of data from the city of Vinhedo. 
We close this section with statistics summarizing the test results and comments on the performances of the different heuristics.

\section{Data Instances}

The full characterization of an instance involves the following parameters: the cardinalities of the sets $V, T$, and $W$; the distance $c$ calculated from the sets $S_{j}, j \in W$; the number $m$; and the parameter $r$ (the maximum difference allowed between the number of nodes in different routes).

In order to observe the behavior of the heuristics with respect to scale, we have designed five classes of problems, where each class is defined by the total of nodes in $V \cup W$ : the first class has $|V|=50,|W|=50$ corresponding to KroA100, KroB100, KroC100, KroD100 and KroE100; the second class $|V|=50,|W|=100$ corresponding to KroA150 and KroB150; the third class $|V|=100,|W|=100$ corresponding to KroA200 and KroB200; the fourth class $|V|=100$, $|W|=218$ corresponding to lin318; and the fifth class $|V|=200,|W|=200$ corresponding to rd400. Each class is subdivided in three subclasses, according to the cardinality of $T$ : the subclass 1 has $|T|=\lceil|V| / 8\rceil$; the subclass 2 has $|T|=\lceil|V| / 4\rceil$; and the last one has $|T|=$ $\lceil|V| / 2\rceil$.

Each instance is characterized by a collection of pairs $\left\{\left(x_{i}, y_{i}\right)|i=1, \ldots| V,|+| W \mid\right\}$, where both $x_{i}$ and $y_{i}$ are interpreted as coordinates of points in the Euclidean plane. The base coordinates are chosen from the collection so that it is centralized. Pairs corresponding to the nodes in $T^{*}$ are chosen sequentially in this list, and are followed by the pairs corresponding to the nodes in $V \backslash T$. All other pairs correspond to nodes in $W$.

Next we choose the constant $c$ used in the definition of the neighborhood $S_{j}$ of node $j \in W$. That is, the nodes in $V$ which are close enough to $j$ in the sense that $j$ will be considered covered if the route contains a node in $S_{j}$. The following considerations guide our choice. We want $\left|S_{j}\right| \geq 2$, for all $j$, so $c$ must be greater than the maximum of the distances $c_{j h(j)}$, for all $j \in W$, where $h(j)$ is the index of the node in $V$ that is the second closest node to $j$. Furthermore, we want that every node in $V \backslash T$ covers some node in $W$, otherwise this node could be eliminated from consideration in a pre-processing run. Thus, we also want $c$ to be greater than $m_{h}$, the distance from node $h$ to $W$, for all $h \in V \backslash T$. The value of $c$ is thus the greatest of the two maximum distances.

The number of routes $m$ varied from 2 to 4 in the classes with 100 and 150 points, from 3 to 5 in the classes with 200 and 318 points, and from 4 to 6 in the class with 400 points. Classes with 100 and 150 points have $r=2$, the classes with 200 and 318 points have $r=3$, and the class with 400 points has $r=4$.

\section{City of Vinhedo Instance}

The Municipal Guard of the city of Vinhedo provided two lists of addresses. The first list, with 101 addresses, corresponded to sites that needed to be visited during rounds, and the second, with 133 addresses, contained the addresses of sites that should be covered. The geographical coordinates of these sites were calculated, as well as the coordinates of all street intersections. 
The geographical coordinates of the Municipal Guard base gave us the base node location. This real instance thus had 102 nodes in $T, 133$ nodes in $W$ and 2496 nodes in $V \backslash T$ (the intersections). Figure 3 shows the distribution of points within the city region.

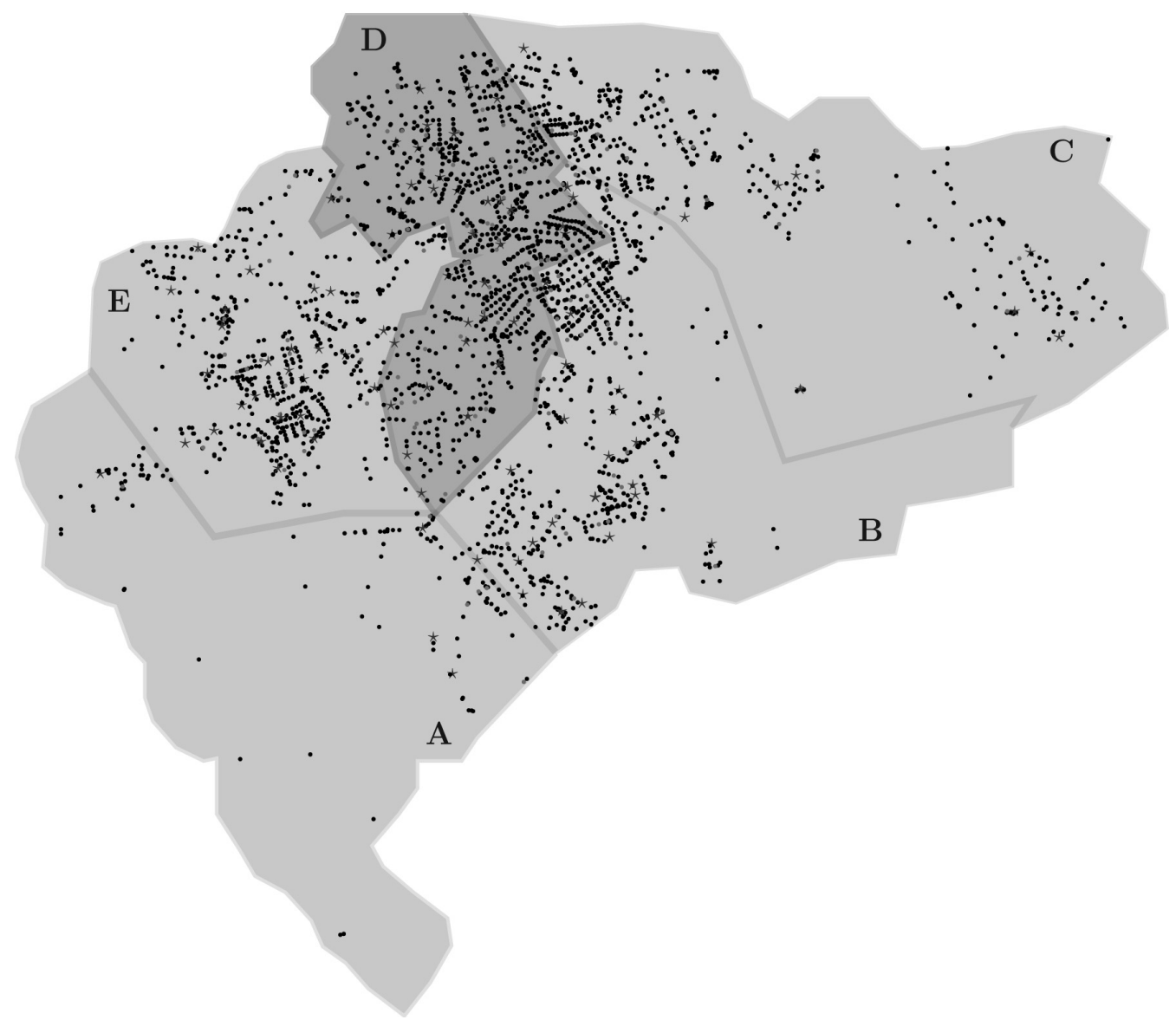

Figure 3 - Distribution of relevant nodes in the City of Vinhedo.

Empirical experience in security acquired by the Municipal Guard leads to the conclusion that a patrolling officer can watch each site up to a distance of $150 \mathrm{~m}$, so we chose $c=150 \mathrm{~m}$. This allowed the elimination of 1563 nodes in $V \backslash T$, whose distances to $W$ were greater than $c$. We verify this by comparing the Figures 3 and 8 . The latter illustrates a set of routes obtained in the tests. Note in Figure 3 that the distribution of nodes in this instance is not uniform, for example, in Sector D there is a higher concentration of nodes than in Sector A. Our real-data instance had $|T|=102,|V \backslash T|=933$ and $|W|=133$. The values considered for $m$ were five, six and seven, which are related to the likely number of cars available for community patrolling duties. The parameter $r$ was chosen as six in the first case $(m=5)$ and as eight in the remaining cases. According to the Municipal Guard this choice does not overload the patrolling officers. 


\section{Summary of Results for TSPLIB Instances}

Figure 4 shows a typical solution of the problem. Notice that the three routes contain all nodes in $T$ (the star shaped nodes), but some nodes in $V \backslash T$ (bullets) were not picked. The gray disks are the neighborhoods of the nodes in $W$ (circles). One can verify that the solution is feasible with respect to coverage, as well as each gray disk contains at least one visited node. Furthermore, the routes are balanced, differing by at most one node.

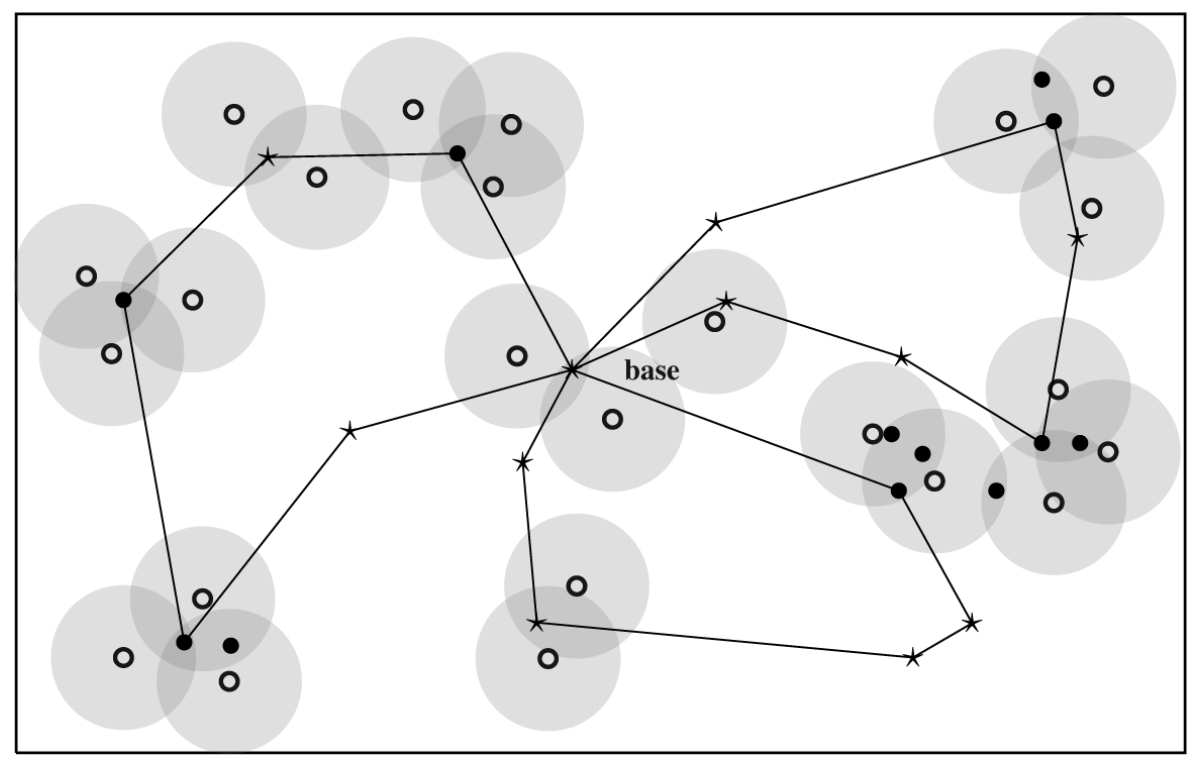

Figure 4 - Example of a feasible solution.

Tables 2, 3 and 4 summarize the relevant data collected in the numerical experiments with the four heuristics listed in Table 1. The first column indicates the instance, the second column the number of vehicle, and for each heuristic we report the $\tilde{r}$ (the maximum difference obtained between the number of nodes of different routes in the approximate solution), the time (in seconds) spent on each instance, the cost (total length of all tours) and the quality index (Q.I.) of the heuristic. This last number is the cost divided by the minimum cost over all four heuristics, showing how good the performance of that heuristic was, as compared to the one with the best cost. Therefore, the Q.I. of the routine with the best cost is 1 , and the table cell containing this entry is shaded for emphasis.

For instances up to 150 points, Sector Partition has the biggest number of the Q.I. equals to 1, approximate $71 \%$ of total, followed by Route-first/Cluster-second with $60 \%$ of total. The worst Q.I. is 1.377 (37.7\% worse than the best result), achieved by the Greedy Selection heuristic, and the average value of Q.I. is 1.033 . We conclude that the performances of the various heuristics with respect to the quality aspect were very similar. 


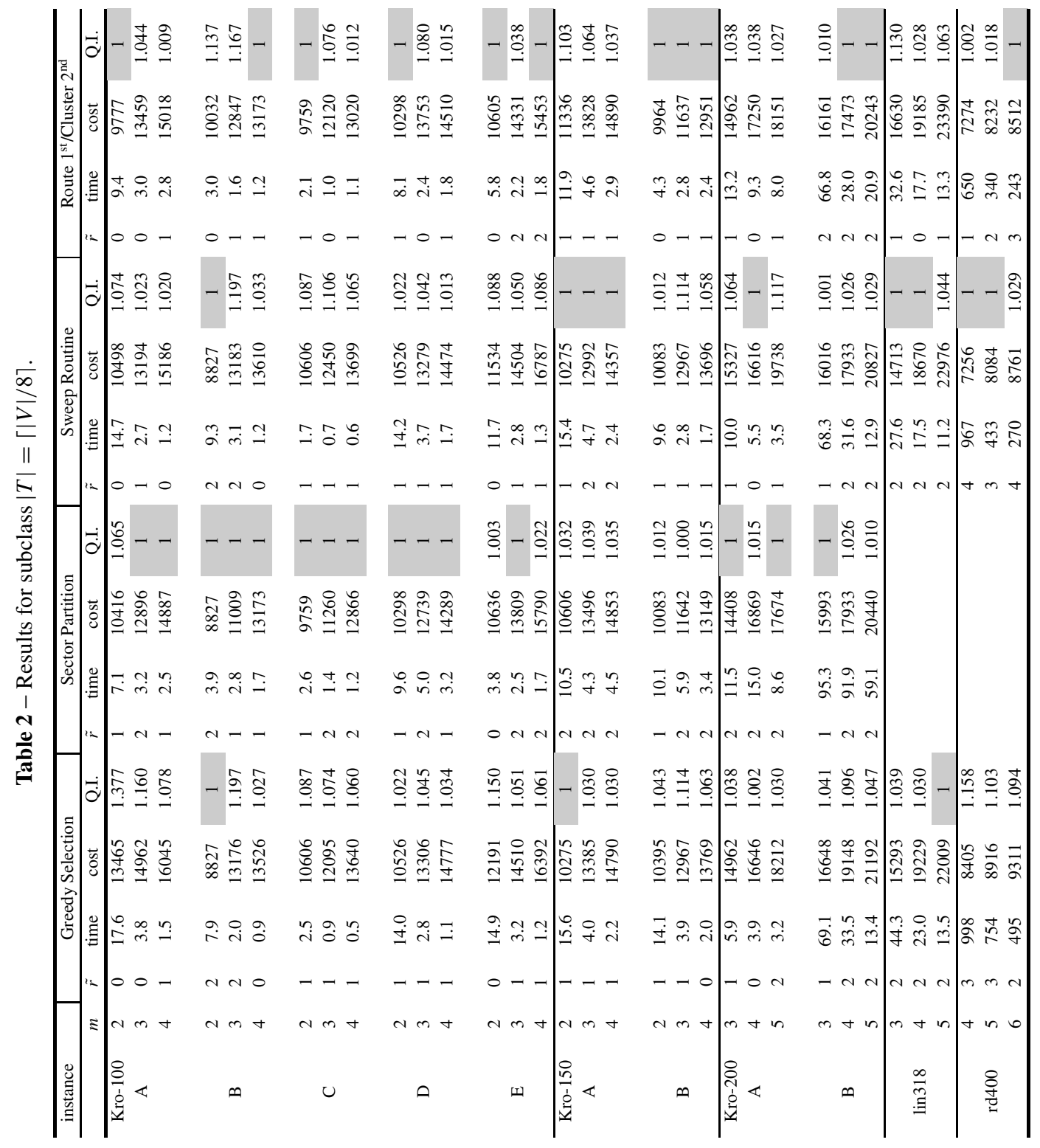




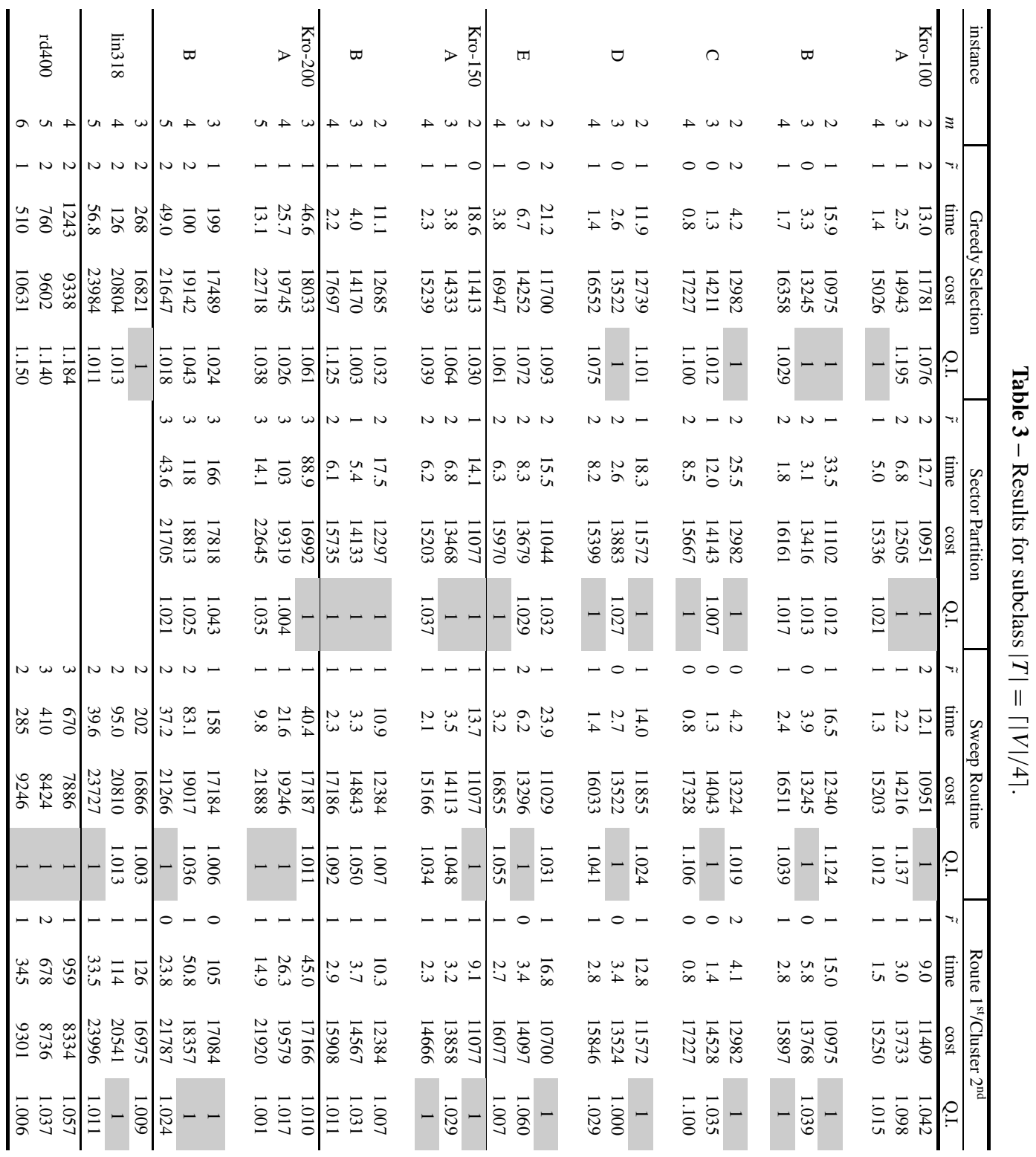




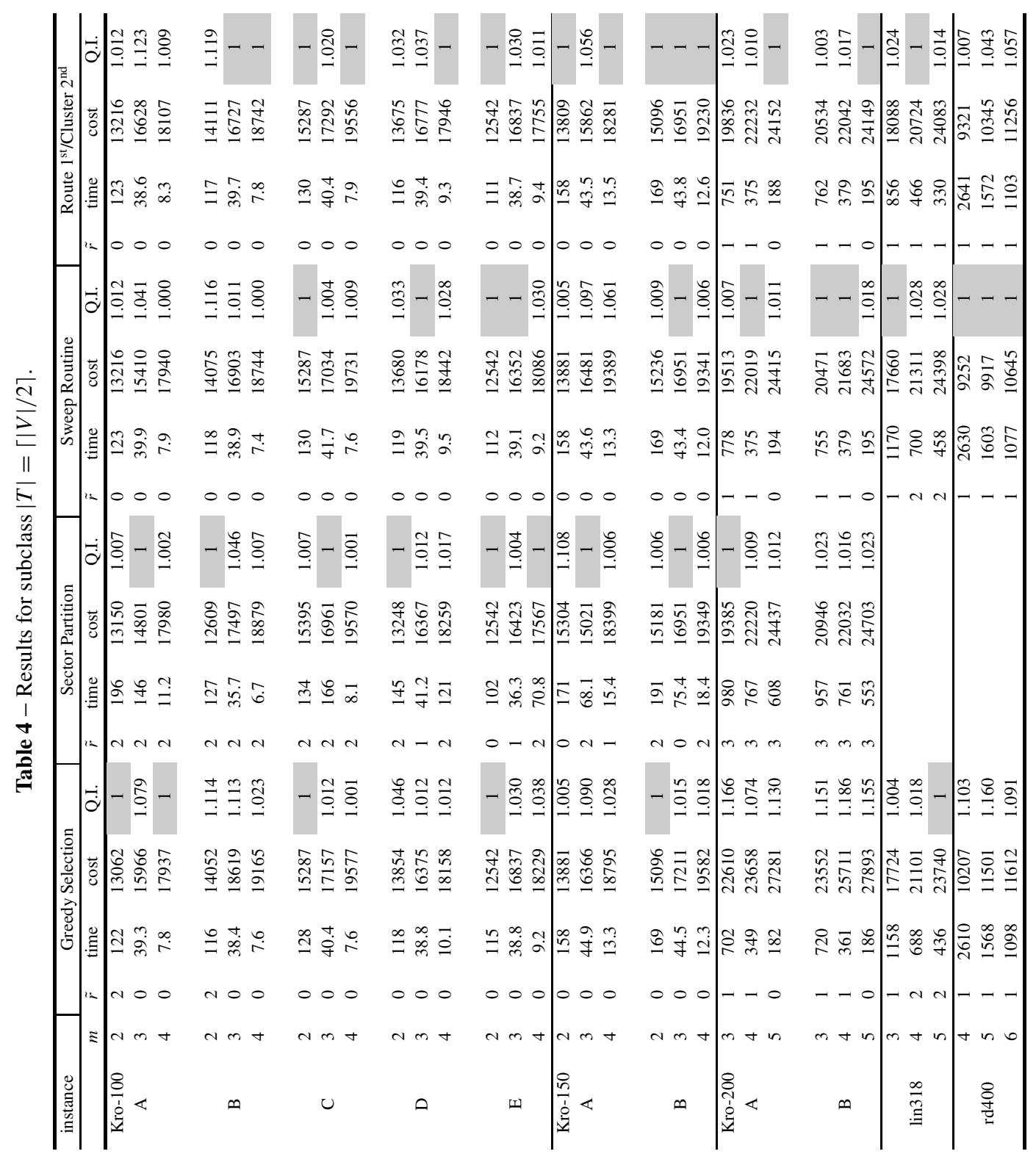




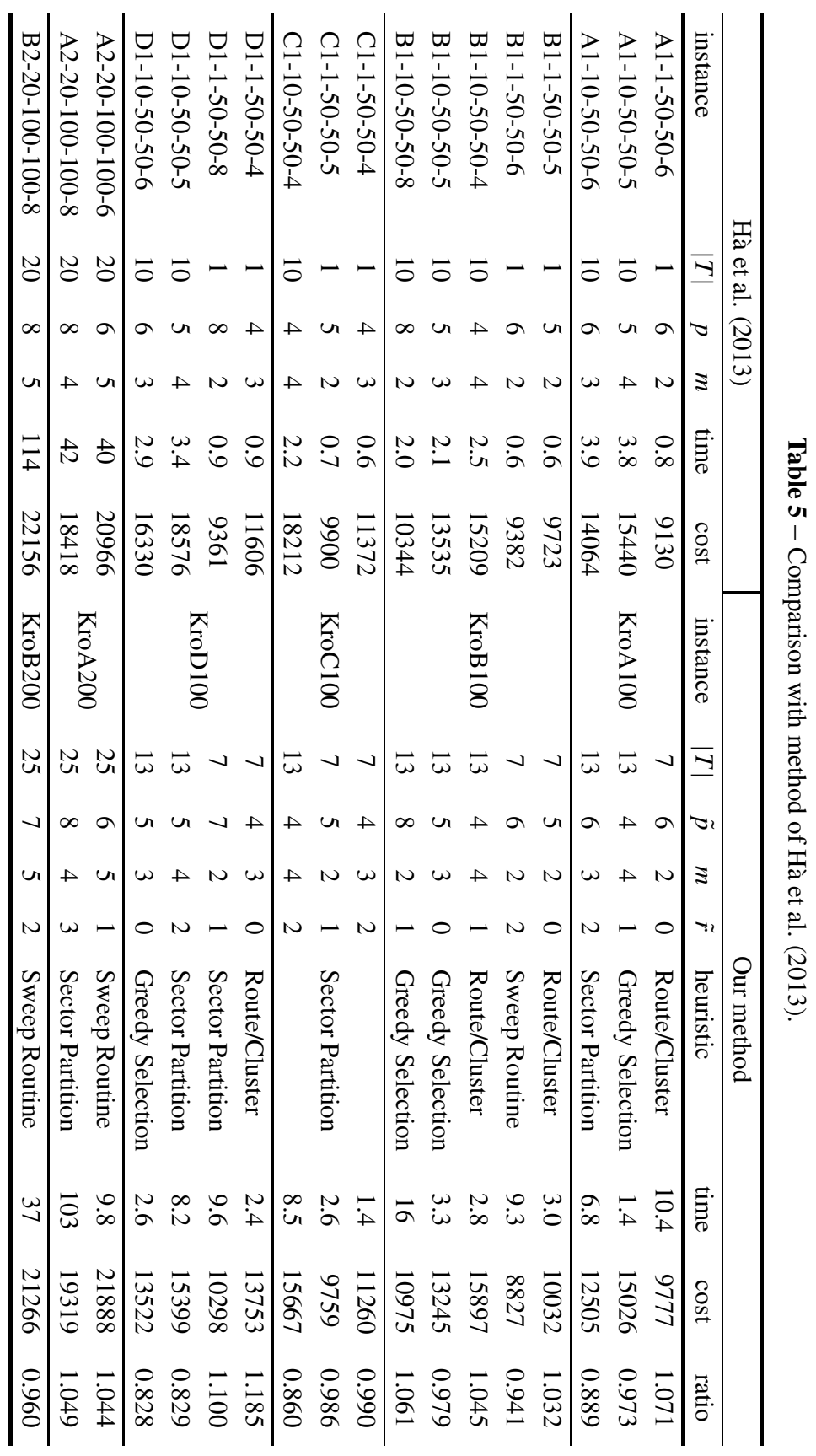


For remaining instances, Sweep Routine has the biggest number of the Q.I. equals to 1, approximate $53 \%$ of total. Since the distribution of points in the instances with 318 and 400 points is non-uniform, the Sector Partition could not produce balanced routes in those instances. And the average value of Q.I. is 1.032, which again indicates similar performances with respect to the cost.

We observed discrepancies in the computational effort (measured by the average time spent) of the heuristics, as illustrated by the charts in Figures 5 and 6. The disparity of ranges led to the construction of two distinct charts. Figure 5 contains data relative to subclasses 100-1 to 150-3, the data of the remaining subclasses are in Figure 6.

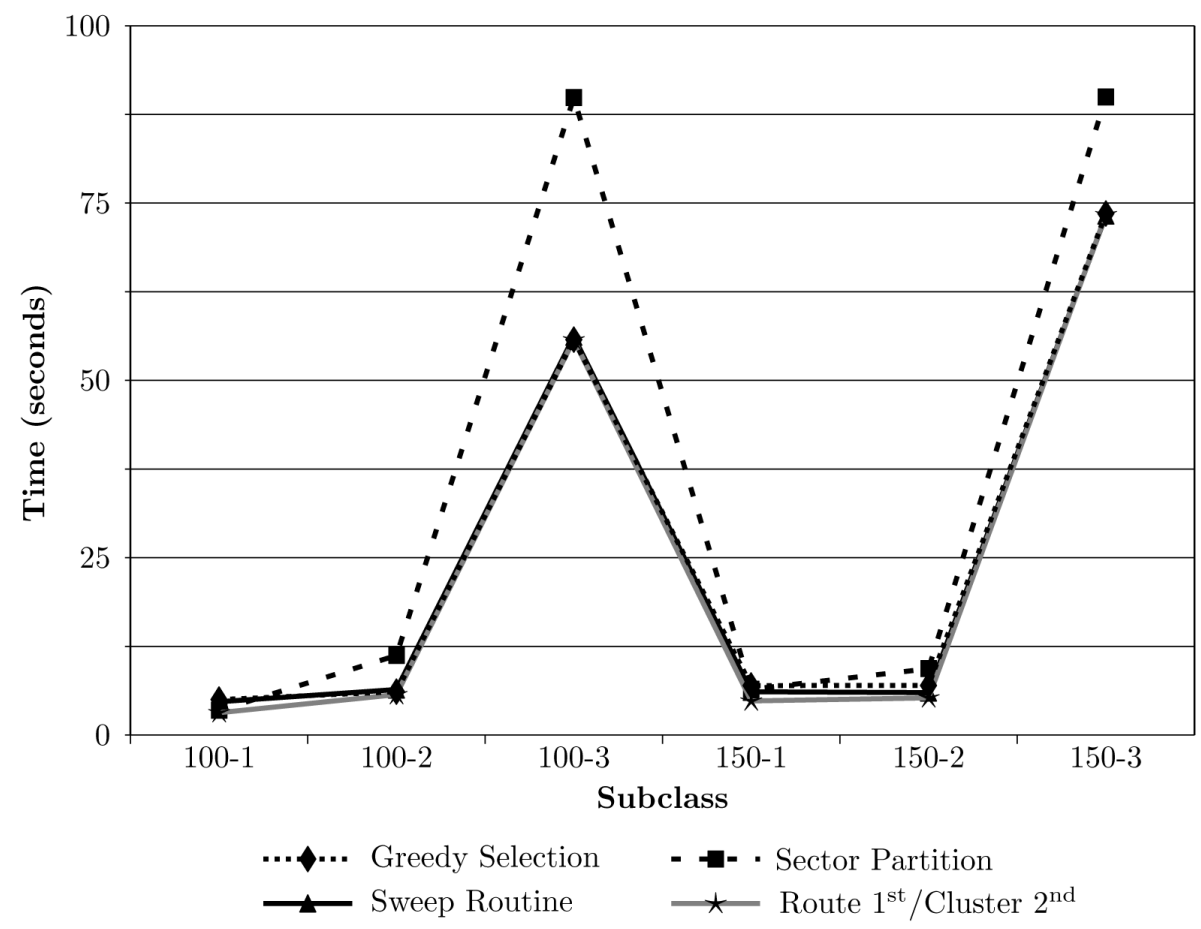

Figure 5 - Average times for subclasses 100-1 to 150-3.

In the first set of subclasses, the Route-first/Cluster-second is the fastest, except for the subclass 150-3, where Sweep Routine does slightly better. This pattern is maintained as the size increases, with the exception of class $400-2$, where its time is $45 \%$ bigger than the minimum time.

This analysis shows that the Sector Partition is well-adapted to smaller instances, where points are uniformly distributed. For larger instances the Sweep Routine obtained solutions with better cost. In terms of speed, the Route-first/Cluster-second obtained better computation times overall, but the performances of all heuristics were very similar in this aspect.

Next, we make a comparison between our results and the ones obtained in Hà et al. (2013), which considers the closest problem variation found in the literature. The main differences between the 


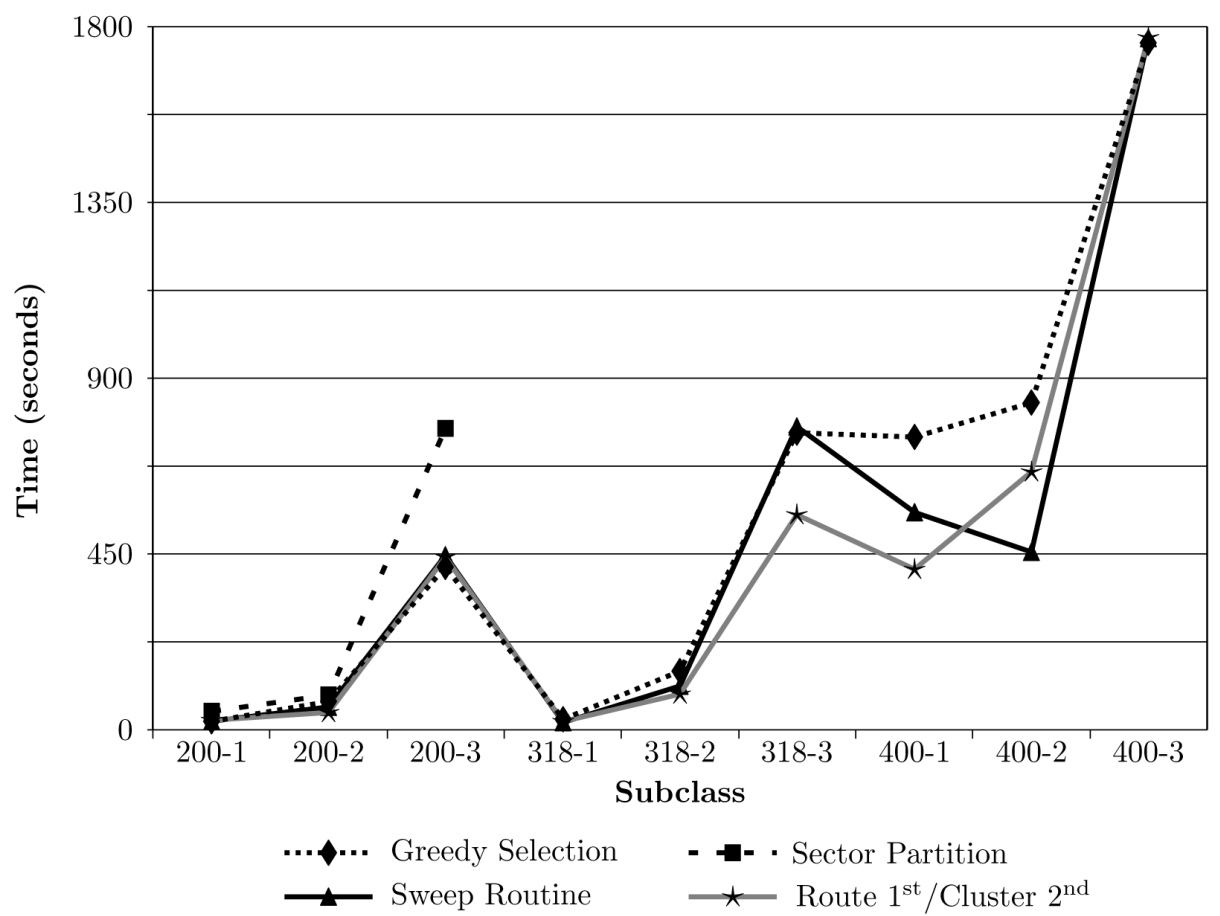

Figure 6 - Average times for subclasses 200-1 to 400-3.

formulations are that in theirs, the number of routes is not fixed, there is a constraint for the number of visits allowed on each route, and the routes may not be balanced.

In order to make a fair comparison, we consider instances in which the number of vehicles in the solution is the same in both approaches, and the maximum number of visits in our solution does not exceed their value.

The results are summarized in Table 5, which is divided into two parts, the first part contains the results from literature, namely the name of the instance, the cardinality of $T$, the maximum number $p$ of points allowed on each route, the number of vehicles, the computational time and the cost. The second part contains the our results for the same instances from TSPLIB, which satisfy the proposed conditions for the comparison. This part contains the name of the instance, $|T|$, the maximum number $\tilde{p}$ of points obtained on each route, $m$, the value of $\tilde{r}$ (the maximum difference obtained between the number of nodes of different routes in the solution), the heuristic considered, time, cost, and the ratio of the cost of our heuristic to theirs.

Analyzing the table we can notice that most of the costs obtained in our solutions were very close to the ones obtained in Hà et al. (2013), and the ratio of all instances varied between 0.828 and 1.185. However, it is important to emphasize that even though we are comparing the same instances from TSPLIB, it was not possible to reproduce the choices of $T, V \backslash T$ and $W$, since only the number of points in each set was provided in their paper. Another point worth noting 
Table 6 - Results from the Vinhedo instance.

\begin{tabular}{rccccll}
\hline \multicolumn{1}{c}{$\begin{array}{c}\text { Cost } \\
(\mathrm{Km})\end{array}$} & Q.I. & $\begin{array}{c}\text { Time } \\
(\mathrm{sec})\end{array}$ & $r$ & $\tilde{r}$ & Vehicles & \multicolumn{1}{c}{ Heuristic } \\
\hline 106.271 & 1.12099 & 55024 & 6 & 3 & 5 & Greedy Selection \\
94.801 & 1 & 50590 & 6 & 2 & 5 & Sweep Routine \\
99.486 & 1.04942 & 41759 & 6 & 3 & 5 & Route 1 st/Cluster 2 $^{\text {nd }}$ \\
134.857 & 1.42253 & - & 6 & 18 & 5 & Empirical \\
\hline 114.022 & 1.08799 & 38887 & 8 & 4 & 6 & Greedy Selection \\
104.800 & 1 & 36110 & 8 & 3 & 6 & Sweep Routine \\
104.806 & 1.00006 & 27084 & 8 & 1 & 6 & Route 1 st/Cluster 2 $^{\text {nd }}$ \\
140.735 & 1.34289 & - & 8 & 35 & 6 & Empirical \\
\hline 121.494 & 1.10100 & 25013 & 8 & 4 & 7 & Greedy Selection \\
110.349 & 1 & 23913 & 8 & 4 & 7 & Sweep Routine \\
111.234 & 1.00802 & 18670 & 8 & 2 & 7 & Route 1 ${ }^{\text {st/Cluster 2 }}{ }^{\text {nd }}$ \\
147.700 & 1.33848 & - & 8 & 35 & 7 & Empirical \\
\hline
\end{tabular}

is that there is no guarantee that their solutions are balanced. Finally, the proximity of the costs obtained in both formulations can be used to measure the quality of our heuristics.

\section{Vinhedo Instance: Comparison of Heuristics}

In practice, a patrolling car is assigned to a geographical area, and the patrolling officers know the locations of the visits and the points that must be observed in this area. More precisely, due to the non-uniform distribution of the geographical points, whenever the number of available patrolling cars is five, two of them are assigned to Sectors A, B and C, two cars are assigned to Sector D, and one car to Sector E. If six cars are available, one car is assigned to each of the sectors A, B, C and E, and two cars to Sector D. Finally, if seven cars are available, one car is assigned to each of the sectors A, B, C and E, and three cars to Sector D. After interviewing the patrolling officers, we noticed that given a geographical sector and set of points to be visited, the officers generally choose their routes according to a greedy rule (Empirical routes), where the next point to be visited is the nearest from the current one, which may be unproductive.

Table 6 reports the cost (in kilometers), quality index (Q.I.), time (in seconds), the value chosen for the parameter $r$, the value $\tilde{r}$ obtained, the size of the fleet, and the heuristic considered together with the empirical routes used by the patrolling officers. The numerical experimental data are collected in three cases: fleet of size five, six or seven.

The Sector Partition heuristic is omitted from Table 6 because it did not obtain balanced feasible solutions, due to the extremely non-central location of the base of operations. Recall that in the adapted instances from TSPLIB, the base coordinates was chosen from the collection data so that the base was centralized. Thus, in this case, the partition produced by the heuristic obtained a set of balanced routes. 
Table 7 - Comparative between Sweep Routine and Empirical Routes.

\begin{tabular}{lcclcc}
\hline & \multicolumn{2}{c}{ Sweep Routine } & & \multicolumn{2}{c}{ Empirical } \\
\cline { 2 - 3 } \cline { 5 - 6 } & Cost $(\mathrm{Km})$ & Visits & & Cost $(\mathrm{Km})$ & Visits \\
\hline Route 1 & 22.3154 & 37 & & 29.3413 & 33 \\
Route 2 & 22.9958 & 39 & & 38.9261 & 47 \\
Route 3 & 15.0461 & 37 & & 15.9927 & 29 \\
Route 4 & 17.3789 & 39 & & 24.0204 & 39 \\
Route 5 & 17.1356 & 37 & & 26.5766 & 40 \\
\hline Average & 18.9744 & & 26.9714 & \\
\hline
\end{tabular}

Unfortunately, this did not happen in the Vinhedo instance. The geographical sites considered fit in a rectangular area of aspect ratio 1.5, that is, the width to height ratio (see Fig. 8, which shows the routes built by the Sweep Routine heuristic in the 5-vehicle case). If we were to consider the "base region" adopted for the TSPLIB instances, the base of operations should be located in a rectangle of sides $30 \%$ of the bigger rectangle, with same center. In the Vinhedo instance the base lies below and outside this ideal rectangle, missing the region by $6 \%$ of the vertical dimension.

Furthermore, the geographical distribution of sites that need to be visited or covered is highly non-uniform. If we divide the shaded rectangle of Figure 8 in four rectangles by drawing vertical and horizontal lines intersecting at the base, we will find $75.3 \%$ of all nodes concentrated in the northwest rectangle, $15.2 \%$ in the northeast, $6.8 \%$ in the southwest and only $2.7 \%$ in the southeast rectangle. As a result, the Sector Partition heuristic produced quite unbalanced routes, with the maximum difference obtained between two routes exceeding the value of the parameter $r$.

The speed exhibited by the Sector Partition heuristic suggests that it is worthwhile to further investigate its possibilities. One direction for future research is that of a non-uniform division in sectors, choosing the central angles in such a way that the number of nodes in each sector is approximately equal.

Compared with the artificial instances, the bad performance of the Greedy Selection heuristic was confirmed in the (very large scale) Vinhedo instance, with the highest Q.I. in every test considered. However, the Empirical routes obtained by the Vinhedo police were worse than any of the heuristics considered. Figure 7 illustrates the solution for five cars, where two cars are responsible for Sectors A, B and C, two cars are assigned to Sector D, and one car is responsible for Sector E. Considering the overall length of the routes in the first two columns of Table 6, the Empirical routes were about $33 \%$ to $42 \%$ worse than the Sweep Routine (see a example of a solution for five vehicles in Fig. 8). When compared with the Greedy Selection, the Empirical routes were about $21 \%$ to $27 \%$ worse.

In discordance with the previous tests, the Sweep Routine scored better objective function values, although not by much, than the Route-first/Cluster-second heuristic, in all cases considered, as indicated by the shaded cells in Table 6. However, the Sweep Routine exhibited $13 \%$ to $25 \%$ larger times than Route-first/Cluster-second. 


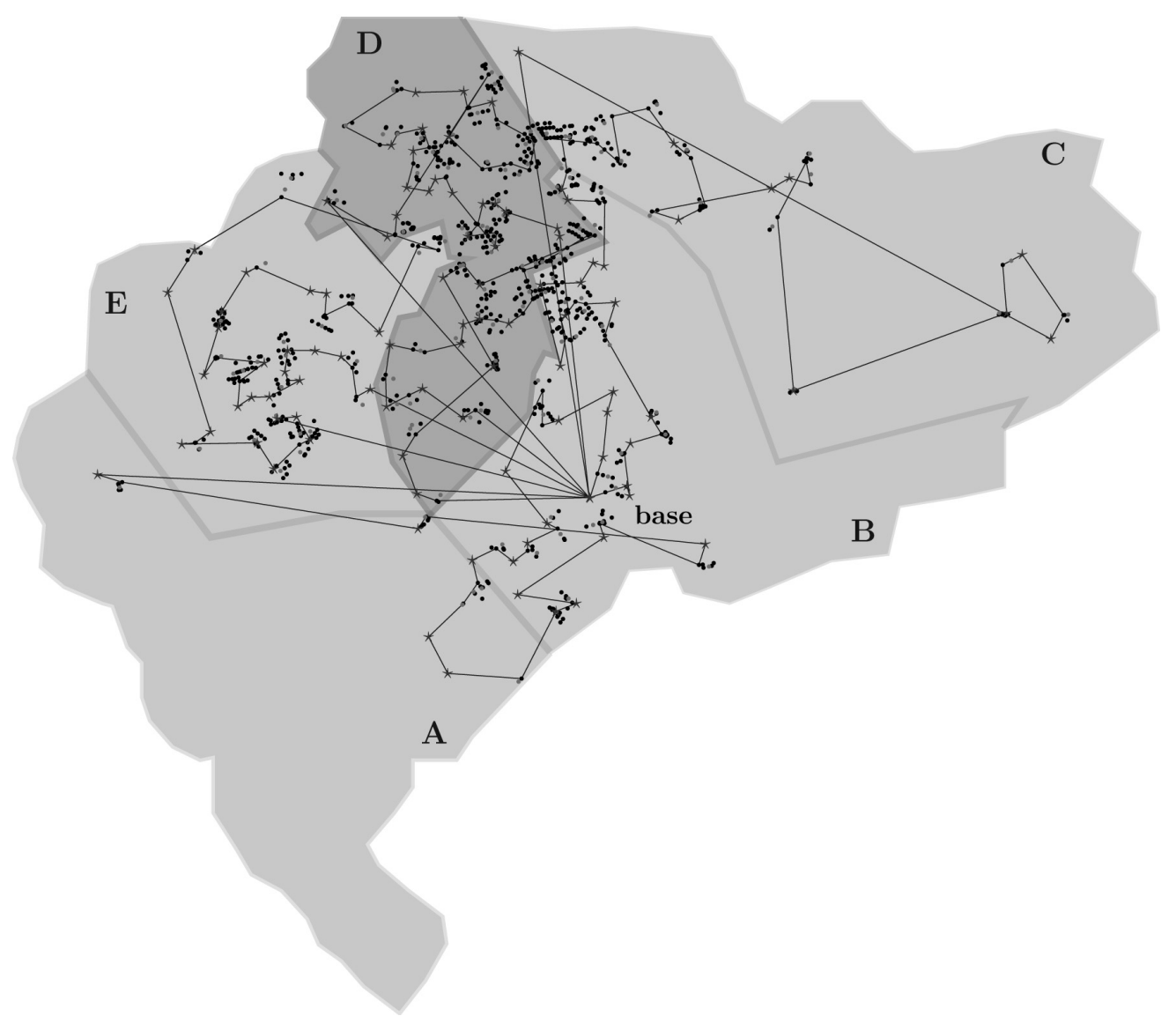

Figure 7 - Routes obtained empirically by patrolling officers.

Since the geographical points of interest are more concentrated in some sectors, the Empirical routes were very unbalanced, which can be seen in the fifth column of Table 6 . Note that there are routes differing by 35 nodes. Moreover, in the case of five vehicles, Table 7 shows the length and number of visits for each individual route. There is a difference of up to 18 visits and the average route lengths in the Sweep Routine is about $30 \%$ lower.

The solutions obtained were considered acceptable since a patrolling car may cover this distance several times during a work shift. This contemplates very well the objective of providing a good coverage with the available resources.

\section{CONCLUSIONS AND FUTURE WORK}

We developed, implemented and tested various heuristics for the construction of routes in the context of urban patrolling. They were compared in numerical tests involving a set of adapted instances from TSPLIB, and variants of a problem defined with real data from the city of Vinhedo, Brazil. 


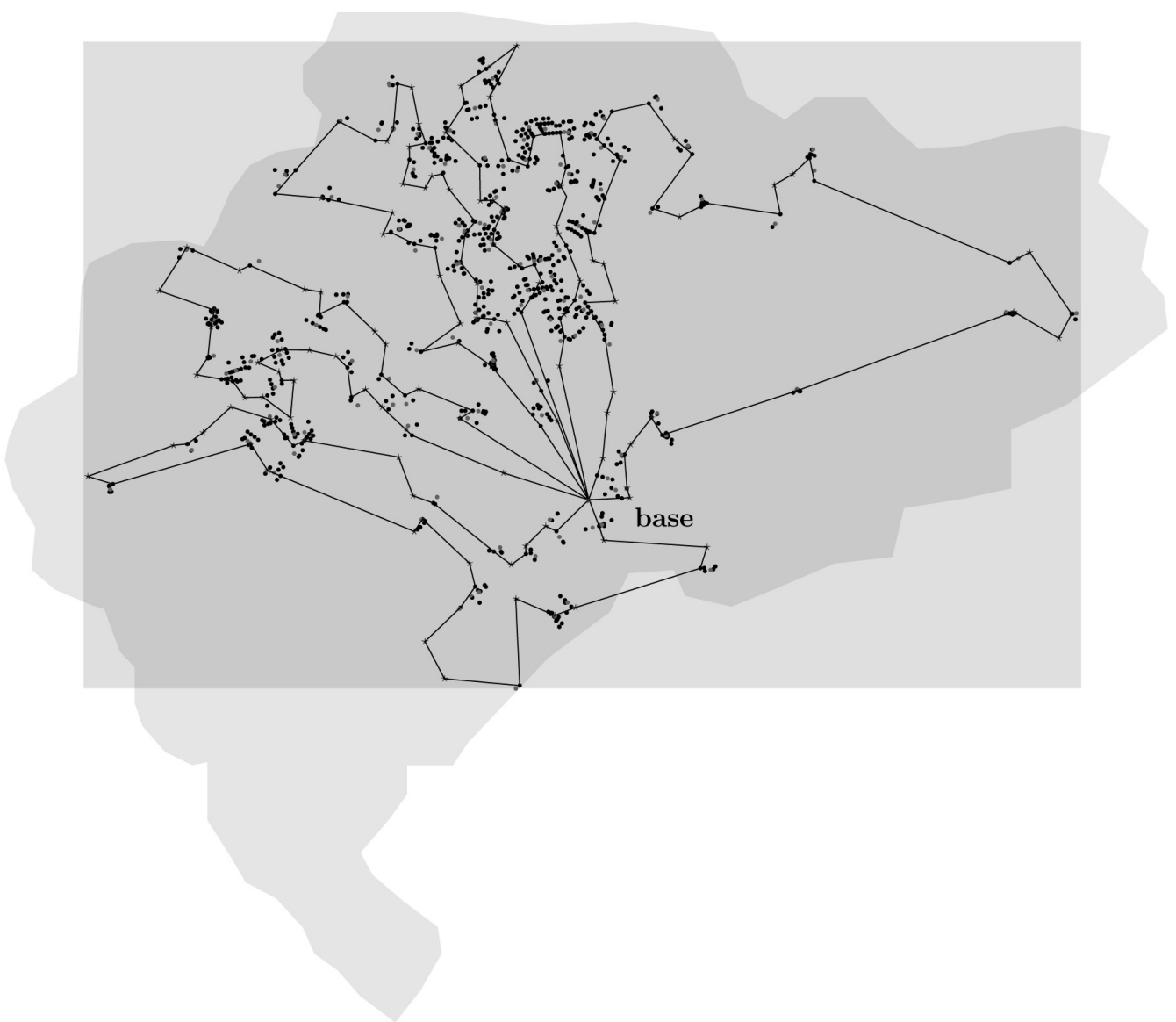

Figure 8 - Routes obtained by the Sweep Routine for the Vinhedo instance.

We developed a modified Sweep Routine, which produced solutions with lower costs for larger instances, while the Sector Partition heuristic, developed by us produced solutions with lower costs for smaller instances (up to 150 points). The computational time was quite uniform for all heuristics.

The heuristics were tested using the Vinhedo instance. The Greedy Selection heuristic, when compared with the other heuristics, produced solutions with lower quality. The solutions of our modifications of the Sweep Routine and Route-first/Cluster-second Routine had similar quality, though the former was always better. On the other hand, the latter was consistently faster.

The solutions obtained in the Vinhedo instance show the advantage of using this kind of modeling and heuristics for large scale problems, since the numerical results show that the Empirical routes used by the Municipal Guard are not balanced, interfering with the contact between the patrolling officers and the members of the community, which is the main objective of the community policing. We conclude that the division in sectors used by the Guard can be improved by using our solutions (see Figs. 7 and 8). 
We observed that the Sector Partition heuristic needs improvement in order to be applied in real instances having highly non-uniform distribution of nodes, turning it into a competitive and robust routine. This will be the subject of future research.

\section{ACKNOWLEDGMENTS}

The first author was partially support by CAPES and FAEPEX (grant 534/09). The second author was partially supported by CNPq-PRONEX Optimization and FAPESP (grant 2006/53768-0). The authors thank Osmir Cruz, Commander of the Municipal Guard of Vinhedo, the municipal government of Vinhedo and the company SSR-Tecnologia for translating the list of addresses into a list of geographical coordinates.

\section{REFERENCES}

[1] BALAS E \& Ho A. 1980. Set covering algorithms using cutting planes, heuristics, and subgradient optimization: A computational study, in PAdberg M., (Ed.), 'Combinatorial Optimization', Vol. 12 of Mathematical Programming Studies, Springer Berlin Heidelberg, pp. 37-60.

[2] Baldacci R, Boschetti M, Maniezzo V \& Zamboni M. 2005. Scatter search methods for the covering tour problem, in Sharda R, Voss S, Rego C \& Alidaee B., (Eds.), 'Metaheuristic Optimization via Memory and Evolution', Vol. 30 of Operations Research/Computer Science Interfaces Series, Springer US, pp. 59-91.

[3] BROWN M \& FINTOR L. 1995. US screening mammography services with mobile units: results from the national survey of mammography facilities. Radiology, 195(2): 529-532.

[4] FoORD F. 1995. Gambia: evaluation of the mobile health care service in West Kiang district. World health statistics quarterly. Rapport trimestriel de statistiques sanitaires mondiales, 48(1): 18-22.

[5] Gendreau M, Hertz A \& Laporte G. 1992. New insertion and postoptimization procedures for the traveling salesman problem. Operations Research, 40(6): 1086-1094.

[6] Gendreau M, Laporte G \& Semet F. 1997. The covering tour problem. Operations Research, 45(4): $568-576$.

[7] HÀ MH, Bostel N, Langevin A \& Rousseau LM. 2013. An exact algorithm and a metaheuristic for the multi-vehicle covering tour problem with a constraint on the number of vertices. European Journal of Operational Research, 226(2): 211-220.

[8] Hachicha M, Hodgson MJ, Laporte G \& Semet F. 2000. Heuristics for the multi-vehicle covering tour problem. Computers \& Operations Research, 27(1): 29-42.

[9] Helsgaun K. 2009. General k-opt submoves for the Lin-Kernighan TSP heuristic. Mathematical Programming Computation, 1(2-3): 119-163.

[10] Hodgson MJ, Laporte G \& Semet F. 1998. A covering tour model for planning mobile health care facilities in Suhum District, Ghama. Journal of Regional Science, 38(4): 621-638.

[11] JOZEFOWIEZ N. 2014. A branch-and-price algorithm for the multivehicle covering tour problem. Networks, 64(3): 160-168. 
[12] Jozefowiez N, SEMET F \& TALBI E. 2004. A multi-objective evolutionary algorithm for the covering tour problem. Applications of multi-objective evolutionary algorithms, COELLO CA \& LAMONT GB, (Eds.), World Scientific, pp. 247-267.

[13] Jozefowiez N, Semet F \& Talbi E. 2007. The bi-objective covering tour problem. Computers \& Operations Research, 34(7): 1929-1942.

[14] LABbÉ M \& LAPORTE G. 1986. Maximizing user convenience and postal service efficiency in post box location. Belgian Journal of Operations Research Statistics and Computer Science, 26: 21-35.

[15] LAPORTE G. 1992. The vehicle routing problem: An overview of exact and approximate algorithms. European Journal of Operational Research, 59(3): 345-358.

[16] Lenstra JK \& Rinnooy KAN AHG. 1975. Some simple applications of the travelling salesman problem. Operational Research Quarterly, 26(4): 717-733.

[17] Lin S \& Kernighan BW. 1973. An effective heuristic algorithm for the traveling-salesman problem. Operations Research, 21(2): 498-516.

[18] Lopes R, Souza VAA \& Cunha AS. 2013. A Branch-and-price Algorithm for the Multi-Vehicle Covering Tour Problem. Electronic Notes in Discrete Mathematics, 44: 61-66.

[19] Motta L, Ochi L \& Martinhon C. 2001. Grasp metaheuristics for the generalized covering tour problem, in 'Proceedings of IV metaheuristic international conference, Porto, Portugal', Vol. 1, Citeseer, pp. 387-93.

[20] NEV/USP. 2009. Manual de policiamento comunitário: polícia e comunidade na construção da segurança, Brasília: Núcleo de Estudos da Violência da Universidade de São Paulo; SEDH, São Paulo.

[21] OliveIRA WA. 2008. Construção de rotas para patrulhamento urbano preventivo, Master's Thesis, Institute of Mathematics, Statistics and Scientific Computing, Department of Applied Mathematics, State University of Campinas, Campinas, Brazil.

[22] OpPong JR \& Hodgson MJ. 1994. Spatial accessibility to health care facilities in Suhum District, Ghana. The Professional Geographer, 46(2), 199-209.

[23] SImms JC. 1989. Fixed and mobile facilities in dairy practice. The Veterinary Clinics of North America - Food Animal Practice, 5(3): 591-601.

[24] Swaddiwudhipong W, Chaovakiratipong C, Nguntra P, Mahasakpan P, LerdlukaNAVONGE P \& KoONCHOTE S. 1995. Effect of a mobile unit on changes in knowledge and use of cervical cancer screening among rural Thai women. International Journal of Epidemiology, 24(3): 493-498. 